\title{
Report on the 31st International Florida Artificial Intelligence Research Society Conference (FLAIRS-31)
}

\author{
Keith Brawner, Vasile Rus, \\ Roman Barták, Zdravko Markov
}

\begin{abstract}
The 31st International Florida Artificial Intelligence Research Society Conference (FLAIRS-31) was held May 2123, 2018, at the Crowne Plaza Oceanfront in Melbourne, Florida. The conference events included invited speakers, special tracks, and presentations of papers, posters, and awards. The conference chair was Zdravko Markov from Central Connecticut State University. The program cochairs were Vasile Rus from the University of Memphis and Keith Brawner from the Army Research Laboratory. The special tracks were coordinated by Roman Barták from Charles University in Prague.
\end{abstract}

$\mathrm{T}$ he Florida Artificial Intelligence Society (FLAIRS) was founded in 1987 to promote and advance artificial intelligence research in the state of Florida and to foster the exchange of ideas and collaboration between researchers at the universities and pioneers in industry through the vehicle of an annual conference. Shortly thereafter, the FLAIRS conference, a general AI conference, grew to become a major venue for AI researchers from around the world to present their work. The conference continues its incooperation status with the Association for the Advancement of Artificial Intelligence.

Continuing a long tradition of presenting and discussing state-of-the-art artificial intelligence research in a sociable atmosphere and a beautiful setting, the 31st International Florida Artificial Intelligence Research Society Conference (FLAIRS-31) took place May 21-23, 2018, in Melbourne, Florida. It attracted 235 PC members from 29 countries, with 


\title{
The 32nd International FLAIRS Conference
}

\author{
Lido Beach Resort, Sarasota, Florida, USA \\ May 19-22, 2019 \\ www.flairs-32.info
}

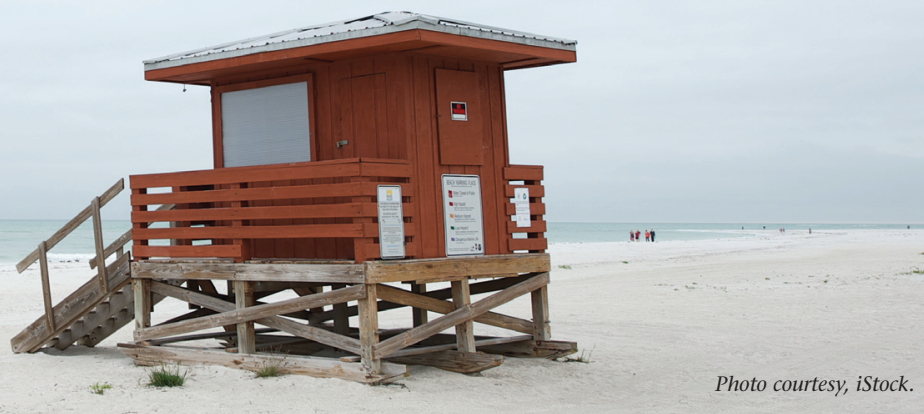

about half coming from outside the US, and 148 attendees from 14 countries with about a third coming from outside the US. The program consisted of a general session spanning a broad range of AI research areas such as machine learning, reasoning, and optimization, and 10 special tracks focused on particular topics in artificial intelligence.

An integral part of the conference, the special tracks provide researchers working in similar areas the opportunity to meet and present work in those areas. These focused sessions also offer forums for interactions within a broader community of AI researchers. The special tracks program included sessions and papers on AI for big social data analysis; AI for games, serious games, and multimedia; AI in healthcare informatics; applied natural language processing; autonomous agents and robots; intelligent learning technologies; recommender systems; uncertain reasoning; case-based reasoning; and semantics, logics, information extraction, and AI.

The call for papers attracted 150 submissions (61 to the general conference and 89 to the special tracks) and 27 poster abstracts. The accepted submissions comprised 70 full papers (24 from the general conference and 46 from the special tracks), 34 short papers presented as posters, and 24 poster abstracts that appeared in the proceedings. The Best Paper award went to Felipe Soares da Costa and Peter Dolog for Hybrid Learning Model with Barzilai-Borwein Optimization for Context-Aware Recommendations. The Best Student Paper award went to Jônatas Wehrmann, Mauricio Lopes, and Rodrigo Barros for Self-Attention for Synopsis-Based Multi-Label Movie Genre Classification. The Best Poster award was presented to Himan Abdollahpouri, Robin Burke, and Bamshad Mobasher for Value-Aware Recommendation with Multiple Stakeholders.

The conference featured a stimulating set of invit- ed talks by three distinguished speakers. Rina Dechter from the University of California at Irvine gave a talk entitled "Probabilistic Reasoning Meets Heuristic Search," Raymond Mooney from the University of Texas at Austin gave a talk called "Robots That Learn Grounded Language Through Interactive Dialogue," and Peter Wurman of Cogitai's talk was "How Kiva Robots Disrupted Warehousing." In addition, the invited speakers to the special tracks were Kallirroi Georgila from the University of Southern California, Institute for Creative Technologies, who talked about challenges in reinforcement learning of negotiation dialogue policies; Lewis Frey from the Hollings Cancer Center, who gave a presentation on artificial intelligence and precision medicine; and Santiago Ontañón, of Drexel University, speaking about similarity assessment for structured representation.

The next FLAIRS conference (FLAIRS-32) will be held May 19-22, 2019, in Sarasota, Florida. Information about FLAIRS-32, including the call for papers, is available online at www.flairs-32.info.

Keith Brawner is a researcher and project manager at the US Army Research Laboratory. His research interests are in the areas of machine learning for educational applications, and cognitive architectures.

Vasile Rus is the William Duanavant Professor of Computer Science at the University of Memphis. His research areas are natural language processing, interactive systems, and data science.

Roman Barták is a full professor of computer science at Charles University. His research areas are intelligent autonomous agents, model-based approaches, automated planning and scheduling, and verifiable AI.

Zdravko Markov is a professor of computer science at Central Connecticut State University. His research areas are machine learning, data and web mining, and AI education. 